\title{
Colaboración Universidad-Empresa y Desarrollo Regional. El caso de Oruro (Bolivia)*
}

\author{
Padilla Meléndez, Antonio** \\ Fuster Martín, Elena***
}

\section{Resumen}

En este trabajo se estudian los factores que determinan la Transferencia de Tecnología y Conocimiento (TT) entre la universidad y la empresa, en un país en vías de desarrollo. Su objetivo es analizar el impacto de estas actividades sobre el desarrollo regional, adoptando la perspectiva de innovación abierta y el enfoque del individuo. Para ello, tras una revisión de la literatura sobre el tema, se ha aplicado la metodología cualitativa, realizándose diez entrevistas personales semi-estructuradas a docentes de la Universidad Técnica de Oruro (UTO) y gerentes de pequeñas y medianas empresas del Departamento de Oruro (Bolivia). Los principales resultados reflejan que estas actividades son todavía muy incipientes en la región, por lo que su impacto en el desarrollo regional es aún escaso. Como conclusiones, este estudio establece que los factores determinantes de las actividades de transferencia entre la UTO y el sector empresarial se pueden dividir en oportunidades y barreras. Entre las oportunidades para la UTO destacan las mejoras internas y un rol más proactivo en la sociedad. Para las empresas son la mejora en la productividad y en la competitividad. Por otro lado, las barreras que dificultan la TT son burocráticas, falta de financiación, falta de confianza y falta de profesionalidad. Por tanto, es clave establecer una política clara que regule las actividades de TT.

Palabras clave: Transferencia de Tecnología y Conocimiento, Desarrollo Regional, Innovación abierta, Países en vía de desarrollo.

\section{Recibido: 18-04-1.3 Aceptado: 01-06-14}

Se agradece la colaboración de la organización Punto de Apoyo (Point d'Appui), de la Universidad Técnica de Oruro y de la Federación de Empresarios Privados de Oruro (FEPO), por facilitar el acceso a los expertos, así como a la Universidad de Málaga por la financiación de la estancia en Bolivia.

** Profesor Titular. Facultad de Estudios Sociales y del Trabajo Universidad de Málaga-España. Email: apm@uma.es

*** Investigadora. Facultad de Estudios Sociales y del Trabajo Universidad de Málaga -España. Email: elena.fuster@yahoo.com 


\title{
University-Business Collaboration and Regional Development. The Case of Oruro, Bolivia
}

\begin{abstract}
This paper explores the factors that determine knowledge and technology transfer (TT) between the university and business in a developing country. The objective is to analyze the impact of these activities on regional development by adopting the open innovation perspective and focusing on the individual. To do this, after a literature review and using qualitative methodology, ten semistructured interviews with teachers at the Technical University of Oruro (UTO) and managers of small and medium enterprises in the Department of Oruro (Bolivia) were conducted. The main results show that these activities are still very incipient in the region, so their impact on regional development is low. In conclusion, this study establishes that the determinants for TT activities between the UTO and the business sector can be divided into opportunities and barriers. Among the opportunities for the UTO, internal improvements and a more proactive role in society stand out. For the companies, the opportunities are improved productivity and competitiveness. On the other hand, the barriers to TT are bureaucratic, lack of funding, lack of confidence and lack of professionalism. Therefore, it is very important that a clear policy be established to regulate technology transfer activities.
\end{abstract}

Key words: knowledge and technology transfer, regional development, open innovation, developing countries.

\section{Introducción}

Debido a la creciente importancia del conocimiento como factor de desarrollo económico, las universidades, como principales agentes de creación de conocimiento, han ido experimentando un cambio en su rol (Etzkowitz et al., 2000; Etzkowitz, 2003). Estas instituciones realizan actividades de docencia e investigación y, además, transfieren los resultados al exterior para que la sociedad en general pueda beneficiarse los mismos. Las empresas acuden a la universidad buscando el conocimiento que necesitan para ser más innovadoras y más competitivas favoreciendo así el desarrollo regional (Shaw y Allison, 1999). Así, surgen las actividades de Transferencia de Tecnología y Conocimiento (TT) entre universidad y empresa como forma de colaboración y como una herramienta de desarrollo regional.

La mayoría de las investigaciones realizadas en este ámbito reflejan que existen muchos factores que incentivan la transferencia, como, los beneficios monetarios o el reconocimiento dentro de la comunidad científica, entre otros (Debackere y Veugelers, 2005; Siegel et al., 2003b; Siegel et al., 2003a), pero también existen numerosas barreras que la dificultan (Decter et al., 2007; Siegel et al., 2004), lo que pone de manifiesto la necesidad de seguir investigando sobre el tema. Además, no existen trabajos que analicen los factores que afectan a la TT entre la universidad y la empresa en países en vías de desarrollo, aplicando una metodología cualitativa.

Por tanto, este trabajo pretende cubrir esta brecha de investigación anali- 
zando los factores que afectan la TT entre la universidad y la empresa, además de estudiar el impacto de estas relaciones en el desarrollo regional, bajo la perspectiva de la innovación abierta y adoptando un enfoque individual, en países en vías de desarrollo, concretamente en el contexto del Departamento de Oruro (Bolivia). Para ello, las preguntas de investigación que se plantean en este trabajo son:

P.I. 1: ¿Cuáles son los factores que afectan a la transferencia de tecnología y conocimiento entre la universidad y la empresa en el contexto de la innovación abierta y bajo un enfoque individual, en países en vías de desarrollo?

P.I. 2: ¿Cómo afecta la transferencia de tecnología y conocimiento al desarrollo socioeconómico de regiones en países en vías de desarrollo?

Para dar respuesta a las preguntas de investigación planteadas, se ha empleado una metodología cualitativa (Yin, 2011) basada en entrevistas personales a informantes estratégicos del contexto de estudio y siguiendo las recomendaciones establecidas en Bansal y Corley (2012). En concreto, se han realizado diez entrevistas semi-estructuradas en profundidad en la región de Oruro (Bolivia).

La metodología cualitativa se refiere, en su más amplio sentido, a la investigación que produce datos descriptivos: las propias palabras de las personas, habladas o escritas, y la conducta observable (Taylor y Bogdan, 2000), a través de los cuales se permite el análisis de contextos concretos. Los instrumentos utilizados en este trabajo han sido las entrevistas individuales en profundidad y un cuestionario semi-estructurado con 25 preguntas, basado en estudios previos
(Adler y Kwon, 2002; Lockett et al., 2008; Padilla-Meléndez et al., 2013).

Para la selección de los entrevistados, en primer lugar, la Universidad Técnica de Oruro (UTO) proporcionó una lista de siete docentes que mantenían o habían mantenido contacto con el sector empresarial en cuanto a actividades de TT, de los cuales se entrevistó a seis, número relevante considerando que la TT es incipiente en la región de Oruro. En segundo lugar, la Federación de Empresarios Privados de Oruro (FEPO) facilitó el contacto con cuatro empresarios que, además de mantener convenios de colaboración con la UTO, habían participado en la elaboración del contrato establecido entre la FEPO, la UTO y el Gobierno para la creación del primer Parque Tecnológico de la región de Oruro.

De este modo, se realizaron diez entrevistas de forma personal entre septiembre y octubre de 2012. Los nombres empleados en el trabajo son ficticios ya que se garantizó salvaguardar la identidad de los entrevistados.

Una vez procesadas las entrevistas se realizó el análisis de los datos, se comparan cada una de las preguntas y se extraen conclusiones en común (Alvesson y Sköldberg, 2000; Silverman, 2000). Con el objetivo de extraer comunalidades que permitan describir y analizar la situación concreta del Departamento de Oruro, a modo de ejemplo de sistema socioeconómico de un país en desarrollo donde se realiza TT. Con esto, se ha podido comparar los factores motivadores y limitadores de la TT, así como el papel de la universidad en el desarrollo regional, establecidos por la literatura previa, en el caso de la región de Oruro. 
Con el objetivo de mostrar información concreta relevante sobre las opiniones vertidas por los entrevistados.

La contribución principal de este trabajo reside en explicar la relevancia del nivel individual para desarrollar la transferencia en zonas poco desarrolladas, realizando un análisis detallado de los elementos principales de un contexto concreto, en cuanto a oportunidades y barreras.

\section{Impacto de la TT en el desarrollo regional: una revisión teórica}

Al tratarse de un estudio que analiza la transferencia de tecnología y conocimiento (TT) entre la universidad y la empresa bajo la perspectiva de innovación abierta y el impacto de estas actividades sobre el desarrollo regional, es necesario revisar cuidadosamente cada uno de estos conceptos. En primer lugar, se examina el concepto de innovación abierta adaptada a la universidad mediante las actividades de TT, y en segundo lugar, el concepto de desarrollo regional y el vínculo que guarda con la universidad.

\subsection{Innovación abierta y Transferencia}

La innovación ha sido estudiado desde diferentes perspectivas, desde el modelo de adopción de innovaciones de Rogers (1983) hasta el modelo de triple hélice (Etzkowitz, 2003) o el modelo de innovación abierta (Chesbrough, 2003). El modelo de triple hélice es un modelo innovación en espiral, frente al lineal tradicional, que considera la vinculación entre tres órganos institucionales: la universidad, la empresa y el gobierno. Al igual que el modelo de innovación abierta, considera la relación de la organización con su entorno. La principal diferencia entre ambos, es el papel que juega el gobierno en cada uno de ellos, siendo mayor su papel en el caso del modelo de la triple hélice.

La innovación abierta constituye un nuevo paradigma que se define como el uso intencionado de entradas y salidas de conocimiento con el objetivo de acelerar la innovación interna y de ampliar sus mercados externos, respectivamente (Chesbrough et al., 2006). El concepto de innovación abierta aplicada a la universidad y su relación con la empresa a través de la comercialización de sus resultados de investigación ha surgido recientemente como línea de investigación (Gassmann et al. (2010), y en ella se enmarca este trabajo.

La TT es definida como la transferencia de nuevos conocimientos, productos o procesos de una organización a otra para el beneficio empresarial de las partes implicadas (Decter et al., 2007), incluyendo la generación de ideas, creación de spin-offs, licencias de tecnología y propiedad intelectual. Son actividades complejas, interrelacionadas e independientes y con alto riesgo y dinamismo (McAdam et al., 2005). Además, están condicionadas por el sistema de innovación nacional, produciéndose diferencias significativas entre los países.

En este trabajo, los conceptos de transferencia de tecnología y transferencia de conocimiento (TT) se van a utilizar conjuntamente ya que se considera la transferencia de tecnología como la crea- 
ción y asimilación de nuevos conocimientos, lo que, a su vez, lleva implícito el uso de tecnología.

Los agentes involucrados en el proceso de TT son tres: el investigador/científico que descubre nuevas tecnologías, los directivos de las universidades que están relacionados con las empresas y gestionan los derechos de propiedad intelectual, y las empresas/emprendedores que comercializan las tecnologías basadas en la universidad (Siegel et al., 2003b). Para llevar a cabo la TT es necesaria la relación entre estos agentes, relación que puede establecerse mediante mecanismos formales (con un mecanismo jurídico, como contrato, licencias o patentes) o informales (con relaciones no formalizadas).

En cuanto a los factores que determinan la TT, se ha realizado una revisión sistemática de la literatura con el objeto de identificar cuáles son los más comunes (Cuadro 1).

En cuanto a los factores que determinan la TT, en la literatura analizada (Debackere y Veugelers, 2005; Decter et al., 2007; D'Este y Perkmann, 2011; Link et al., 2007; Lockett et al., 2009; Padilla- Meléndez y Garrido-Moreno, 2012; Siegel et al., 2003a, 2003b, 2004) se señala como factores motivadores para los investigadores universitarios beneficios económicos y no económicos. En los económicos se incluyen los beneficios monetarios directos y la obtención de fondos adicionales para la investigación (Debackere y Veugelers, 2005; Decter et al., 2007; Siegel et al., 2003a, 2003b). En los no económicos están el reconocimiento dentro de la comunidad científica (Siegel et al., 2003a, 2003b) y la promoción en la universidad (Link et al., 2007).
Asimismo, se han identificado una serie de factores facilitadores de la TT que ayudan e incentivan al investigador a realizar actividades de TT, entre los cuales se encuentra la necesidad de establecer una estructura de incentivos adecuada para recompensar los esfuerzos de emprendimiento académico (Lockett et al., 2009) y el establecimiento de un servicio centralizado de personal con experiencia en la TT para gestionar los aspectos de formación y forma de contratos que surgen en el proceso de TT (Debackere y Veugelers, 2005).

Por otro lado, los factores que dificultan la TT entre los investigadores son principalmente las barreras culturales e informativas para la TT (Decter et al., 2007; Siegel et al., 2003a, 2003b;), la falta de entendimiento en aspectos normativos entre la universidad y la empresa, las barreras burocráticas y la poca flexibilidad de las instituciones universitarias (Siegel et al., 2004)

\subsection{Desarrollo regional}

El desarrollo económico está cada vez más vinculado a la capacidad de una región para adquirir y aplicar el conocimiento, tendencia que se está viendo acelerada por el proceso de la globalización (Lockett et al., 2009). Cada vez más, las ventajas competitivas de los países vienen determinadas por el grado de innovación y el uso del conocimiento, en vez de por la abundancia de recursos naturales o mano de obra barata. De esta manera, el crecimiento económico puede empezar a ser entendido más como un proceso de acumulación de conocimiento que 


\section{Cuadro 1 \\ Factores que determinan la TT}

\begin{tabular}{|c|c|}
\hline Autor & Factores que facilitan la $\mathrm{T}$ \\
\hline $\begin{array}{l}\text { Siegel et } \\
(2003 a)\end{array}$ & $\begin{array}{l}\text { al., Reconocimiento dentro de la } \\
\text { comunidad científica. } \\
\text { Beneficios monetarios. } \\
\text { Obtención de fondos para } \\
\text { investigación adicional. }\end{array}$ \\
\hline $\begin{array}{l}\text { Siegel } \\
\text { et al.,(2003b) }\end{array}$ & $\begin{array}{l}\text { Reconocimiento dentro de la } \\
\text { comunidad académica a través } \\
\text { del logro de publicaciones. } \\
\text { Beneficios financieros directos. } \\
\text { Obtención de fondos para la } \\
\text { investigación adicional sobre todo } \\
\text { para personal de investigación } \\
\text { y equipo de laboratorio. }\end{array}$ \\
\hline
\end{tabular}

Siegel et al., (2004) No aplicable.
Debackere y

Veugelers

(2005)
Decter et al., Obtención de financiación.

(2007)
Una estructura de incentivos adecuada para recompensar los esfuerzos de emprendimiento académico.
Descentralización de las estructuras de funcionamiento para proporcionar una mayor autonomía a los grupos de investigación.
Un servicio centralizado de personal con experiencia en la TT para gestionar los contratos que surgen en el proceso de TT universidad.

Reclutamiento y selección de personal.

Apoyo de la universidad a la empresa.

Satisfacción con el proceso de TT.

Link et al., (2007) Las variables principales que determinan la TT son:

Remuneración económica del personal investigador.

Recompensas no económicas como la promoción, ascensos o plaza titular.

Relaciones y redes sociales.

Factores institucionales y normas culturales.

Colocación de antiguos alumnos.

El tamaño del grupo de

investigación.
Existencias de barreras culturales e informativas para la TT. Mejora de las capacidades del personal de las oficinas de TT. Más fondos para las oficinas de TT.

Reconocimiento de aquellos que están implicados en la TT y promoción de relaciones informales y redes sociales.

Erradicar las barreras culturales o informativas.

Promover las relaciones informales y las redes sociales.

Desafiar la tradicional apertura científica de la universidad para hacer la transición a la comercialización de la investigación.

El paso de la investigación básica a la investigación aplicada.

La falta de entendimiento en aspectos normativos entre la universidad y la empresa debida a diferencias culturales.

La insuficiente remuneración que reciben al participar en actividades de TT.

La burocracia y falta de flexibilidad de las instituciones universitarias.

No aplicable.

La falta de financiación.

Diferencias culturales.

Problemas de comunicación.

Necesidad de un mayor soporte técnico.

No aplicable. 


\section{Cuadro 1 (Continuación)}

\begin{tabular}{|c|c|c|}
\hline Autor & Factores que facilitan la TT & Factores que limitan Ia TT \\
\hline $\begin{array}{l}\text { Lockett et al., } \\
(2009)\end{array}$ & No aplicable. & $\begin{array}{l}\text { Es necesario un enfoque más estructurado y flexible de las ac- } \\
\text { tividades de transferencia de conocimiento en las universida- } \\
\text { des con el fin de satisfacer las necesidades de los empresarios } \\
\text { para diferentes tipos de apoyos en diferentes momentos para } \\
\text { el desarrollo de sus negocios. }\end{array}$ \\
\hline $\begin{array}{l}\text { D'Este y Perkmann } \\
\text { (2011) }\end{array}$ & $\begin{array}{l}\text { La mayoría de los académicos } \\
\text { colaboran con la industria para } \\
\text { promover su investigación y no para } \\
\text { la comercialización de sus } \\
\text { conocimientos. }\end{array}$ & No aplicable. \\
\hline $\begin{array}{l}\text { Padilla- } \\
\text { Meléndez } \\
\text { y Garrido- } \\
\text { Moreno (2012) }\end{array}$ & $\begin{array}{l}\text { El compromiso del investigador con } \\
\text { la TT está relacionada con el perfil } \\
\text { personal y profesional, el factor } \\
\text { institucional y los factores } \\
\text { de la red social. }\end{array}$ & No aplicable. \\
\hline
\end{tabular}

Fuente: Elaboración propia.

como un proceso de acumulación de capital (O’Shea et al., 2004). Es más, el crecimiento regional no es un fenómeno exógeno $o$ independiente, sino que en parte deriva de la capacidad de las empresas locales para realizar y generar ingresos (Cornett, 2009).

Las universidades, como principales agentes de creación de conocimiento, están ocupando un papel importante en este proceso, experimentando un cambio hacia la "universidad emprendedora" (Etzkowitz et al., 2000; Etzkowitz, 2003) y enfrentándose al reto de evolucionar desde el modelo de "torre de marfil" al de agente de conocimiento (Gassmann et al., 2010). Este cambio en las universidades ha dado lugar a un formato académico empresarial dirigido a satisfacer la necesidad de generar nuevas empresas de conocimiento (Etzkowitz, 2002). Estas empresas, también conocidas como spin-offs, son empresas con fines de lucro basadas en la investigación universitaria (Philpott et al., 2011) y su finalidad es la de estimular el empleo y el crecimiento productivo (Etzkowitz, 2002).

En este sentido, Shaw y Allison (1999) señalan tres formas en las que la universidad puede añadir valor al desarrollo regional. En primer lugar, a través de las spin-offs, pueden contribuir al crecimiento demográfico, a las oportunidades de empleo o al aumento del gasto, entre otros. En segundo lugar, contribuyendo al crecimiento de la economía del conocimiento. En tercer lugar, a través del aprendizaje ofrecen respuestas flexibles e innovadoras a las economías en rápida evolución.

Por su parte, Audretsch et al., (2012); Bercovitz y Feldmann, (2006); Cornett, (2009); Huggins et al., (2012); McAdam et al., (2012) y, Teng, (2010), asumen la universidad como motor de crecimiento económico y como factor importante en el desarrollo regional (Cuadro 2).

Las universidades están reconfigurando su estructura para optimizar su capacidad de innovación (McAdam et al., 2012; 


\section{Cuadro 2 \\ Principales estudios que analizan el papel de la universidad en el desarrollo regional}

\begin{tabular}{|c|c|}
\hline Autor/es & Orientación \\
\hline $\begin{array}{l}\text { Audretsch } \\
\text { et al., (2012) }\end{array}$ & $\begin{array}{l}\text { Analiza cómo la competitividad regio- } \\
\text { nal y los excedentes universitarios } \\
\text { influyen en el comportamiento innovac } \\
\text { de las empresas emprendedoras. }\end{array}$ \\
\hline $\begin{array}{l}\text { Bercovitz } \\
\text { Feldmann } \\
(2006)\end{array}$ & $\begin{array}{l}\text { Analiza el ambiente legal, económico } \\
\text { y las políticas que conforman el sisten } \\
\text { de innovación, y determina } \\
\text { la tasa y el tipo de producción de } \\
\text { conocimiento de la universidad, y por } \\
\text { tanto, } \\
\text { la tasa de cambio tecnológico. }\end{array}$ \\
\hline Cornett (2009) & $\begin{array}{l}\text { Analiza los cambios en las estrategias } \\
\text { de desarrollo y la política puesta en } \\
\text { marcha en Dinamarca en relación con } \\
\text { el desarrollo regional y la innovación. }\end{array}$ \\
\hline $\begin{array}{l}\text { Huggins } \\
\text { et al., (2012) }\end{array}$ & $\begin{array}{l}\text { Analiza la red de contactos de las } \\
\text { organizaciones con las que la } \\
\text { universidad realiza actividades de TT } \\
\text { para ver en qué medida afectan los } \\
\text { factores organizacionales y de } \\
\text { localización. }\end{array}$ \\
\hline
\end{tabular}

McAdam Analiza la relación entre las partes et al.,(2012) interesadas de tres universidades regionales en el contexto de su oficina de transferencia y la Agencia de Desarrollo Regional con el fin de determinar las lecciones aprendidas para el desarrollo de Asociaciones Empresariales Locales emergentes.

Teng (2010) Analiza el nuevo contexto bajo el cual la universidad está empezando a ser vista como un motor de crecimiento socioeconómico y a partir de ahí plantea el modelo de TT entre universidad y empresa desde el punto de vista de la mayoría de los receptores de tecnología en Xi'an (China).

\section{Consideración del papel de la universidad en el desarrollo regional}

Los excedentes universitarios son un factor muy importante para el fomento de la actividad innovadora de las empresas emprendedoras y en la competitividad regional.

El proceso por el cual la universidad dirige el desarrollo económico regional requiere tiempo y paciencia, lo que está a menudo fuera de las demandas inmediatas de los procesos políticos.

La universidad es uno de los actores dentro del proceso de innovación considerando el desarrollo regional como fenómeno endógeno.

La naturaleza y la formación de redes de conocimiento entre las organizaciones con las que la universidad realiza actividades de TT están relacionadas, principalmente, con las características organizacionales de los actores de la red y con su ubicación espacial. Este tipo de redes afecta tanto a la capacidad regional de innovación como a la competitividad regional.

El aumento de la importancia en el desarrollo regional y la economía basada en el conocimiento ha provocado un cambio en el rol de la universidad y su interacción con el mundo empresarial a través de la TT.

Los gobiernos están empezando a ver a las universidades como motores de crecimiento, lo que está animando a éstas a reconfigurar su estructura para optimizar su capacidad de innovación. En este sentido, la relación universidad-empresa mediante las actividades de TT está cobrando importancia como fuente de desarrollo económico regional y como fuente de ingresos para la universidad.

Fuente: Elaboración propia. 
Teng, 2010) y la relación universidad-empresa mediante las actividades de TT está cobrando importancia como fuente de desarrollo económico regional (Teng, 2010). En este sentido, es importante que la universidad establezca redes de conocimiento con las organizaciones, lo cual dependerá de las características organizacionales de los actores de la red y con su ubicación espacial (Huggins et al., 2012). No obstante, para poder conocer el impacto de estas actividades sobre el desarrollo regional se requiere tiempo y paciencia (Bercovitz y Feldmann, 2006), ya que los resultados de la TT entre la universidad y la empresa sobre el desarrollo regional se mide a largo plazo.

Por último, el grado de impacto de la universidad en el desarrollo regional no es igual para todas las universidades. Este depende de: las características internas de la universidad, cómo la universidad responde a los impactos exógenos, la naturaleza de la financiación de las mismas que acompaña al establecimiento de la agenda de los órganos políticos nacionales y regionales en materia de creación de riqueza y las características de la economía regional (Smith y Bagchi-Sen, 2012).

Una vez revisados los antecedentes teóricos de cada uno de los conceptos que intervienen en este análisis, se exponen los resultados obtenidos del análisis cualitativo.

\section{Actividades de transferencia de tecnología y conocimiento en Oruro}

En este apartado se muestran los resultados obtenidos del análisis realizado en la región de Oruro sobre las actividades de TT entre la UTO y las empresas del Departamento, así como los factores que las determinan, y el impacto de éstas sobre el desarrollo regional, siguiendo una metodología cualitativa.

En primer lugar, surge la necesidad de establecer las características del contexto bajo el cual se ha llevado a cabo este trabajo.

Bolivia es un estado plurinacional, descentralizado y con autonomías, estando dividido en nueve departamentos, entre ellos Oruro. En cuanto a las actividades empresariales, la que realiza una mayor aportación al PIB nacional es la industria manufacturera $(17,11 \%)$, seguida de la agricultura, silvicultura, caza y pesca $(13,33 \%)$ y la extracción de minas y canteras (12,21\%) (Instituto Nacional de Estadística de Bolivia, 2010). En cuanto a las empresas, el $95 \%$ son microempresas generando el $84 \%$ del empleo del país (CEPAL y Naciones Unidas, 2012). Entre los problemas generales de las empresas se encuentran: la falta de financiación, la escasa capacitación y asesoramiento empresarial, el escaso acceso a la tecnología y la falta de una normativa específica que apoye al desarrollo de las pequeñas empresas (CEPAL y Naciones Unidas, 2009). Por último, los gastos en actividades científicas y tecnológicas en 2009 constituyeron un $0,16 \%$ del PIB, y la mayoría de las personas dedicadas a estas actividades fueron investigadores que trabajan en universidades como equivalentes a jornada completa (RICYT, 2012). En este sentido, Bolivia se incluye en el grupo de seguidores dinámicos según el índice adelanto tecnológico, lo cual indica que es un país emergente que hace un uso frecuente de las nuevas tecnologías, y que posee conocimientos superiores al 
último grupo de países rezagados según dicho índice (Quiñones y Tezanos, 2011).

El Departamento de Oruro se encuentra situado en la zona del altiplano de Bolivia entre los Departamentos de La Paz y Potosí y compartiendo frontera con la República de Chile. Cuenta con una población aproximada del $4,3 \%$ de la población nacional y una tasa de crecimiento demográfico de $0,72 \%$ (Instituto Nacional de Estadística de Bolivia, 2011). Esta tasa es muy baja debido a la alta tasa de migración existente en el departamento, sobre todo en zonas rurales, hacia otros países (Gobierno Autónomo Departamental de Oruro, 2011). El sector productivo del Departamento está formado prácticamente por actividades del sector primario. El $100 \%$ de los municipios se dedican a actividades relacionadas con la generación de materias primas (Gobierno Autónomo Departamental de Oruro, 2011), destacando como actividades principales la minería, la ganadería y la agricultura, siendo el sector minero el más importante de la región $(36 \%$ del PIB) (Instituto Nacional de Estadística de Bolivia, 2011).

Con respecto a la Universidad Técnica de Oruro (UTO) surge en 1876 pero no es hasta 1941 cuando adopta el nombre actual. Es la universidad más antigua y con mayor trayectoria de la región. Su actividad principal es la formación académica de profesionales, aunque en los últimos años se ha visto ampliada con labores de investigación y de inserción social (UTO, 2009). Los fondos con los que cuenta la UTO están compuestos por la coparticipación tributaria de los impuestos nacionales, la coparticipación tributaria del Impuesto Directo de Hidrocarburos
(IDH) y sus ingresos propios (Gobierno Autónomo Departamental de Oruro, 2011). Cuenta con un Plan de Desarrollo Estratégico para 2009-2013 (UTO, 2009) donde se recogen las líneas estratégicas y objetivos que la UTO pretende conseguir para dicho período. Entre ellos, se encuentra el fomentar la investigación aplicada y el fortalecimiento de la vinculación y las relaciones de la UTO con el exterior, líneas estratégicas directamente relacionadas con la TT.

Ahora bien, los resultados obtenidos se dividen en tres sub-secciones: actividades de TT realizadas, factores que determinan la TT (oportunidades y riesgos) e impacto sobre el desarrollo regional, tal y como se muestran en el Gráfico 1. Para ilustrar las comunalidades encontradas en el análisis, se precisan opiniones literales de los entrevistados, según sean docentes/investigadores (UTO) o empresarios/directivos (PYME).

\subsection{Actividades de Transferencia UTO-Empresas}

Las actividades de TT entre la UTO y las empresas del Departamento de Oruro son todavía muy incipientes, debido principalmente a dos factores: la falta de recursos dirigidos a la investigación y la falta de incentivos por parte de la universidad a los docentes para realizar tareas de investigación. La mayoría de los trabajos de investigación realizados en la UTO han sido producto de iniciativas personales de sus docentes.

En las entrevistas realizadas al personal universitario (Entrevistado 1 al 6 , Entrevista 2012), se indica que están surgiendo numerosas iniciativas dentro de la 


\section{Gráfico 1}

\section{Factores que determinan la TT y el impacto en el desarrollo regiona}

Innovación Abierta

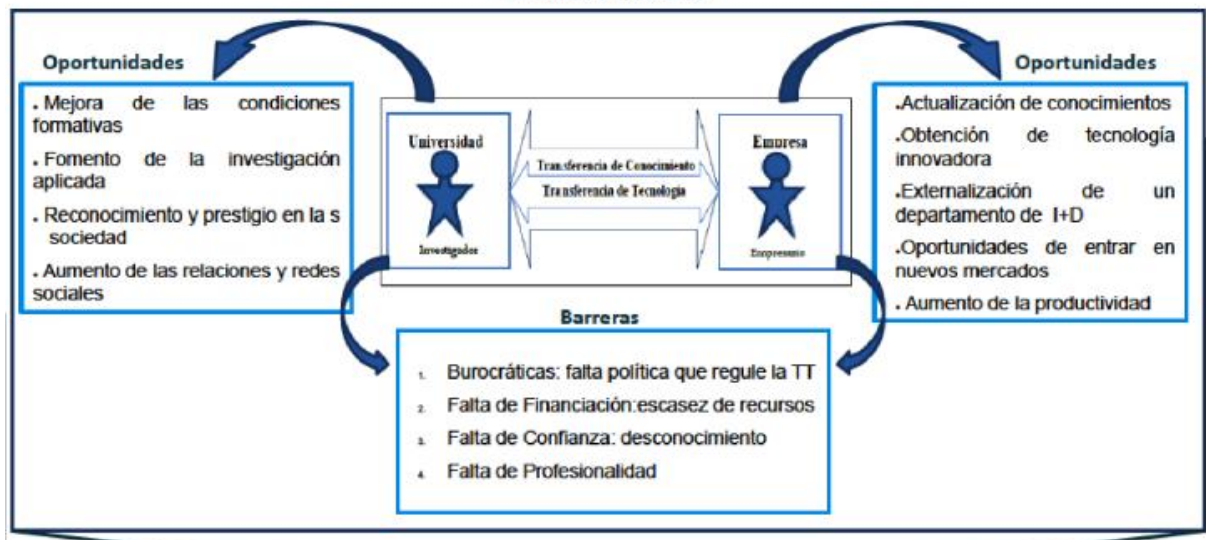

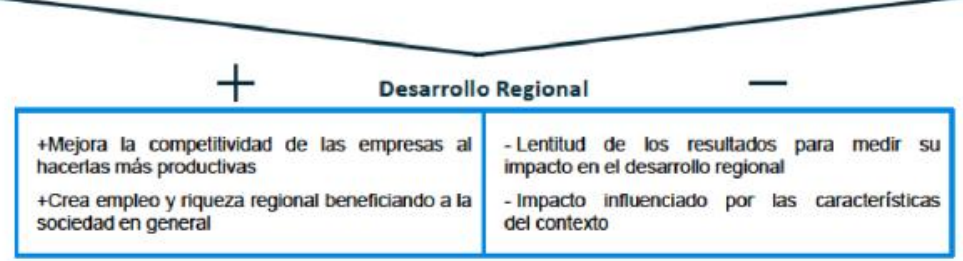

Fuente: Elaboración Propia.

UTO dirigidas a incentivar la investigación universitaria y su difusión hacia el exterior. Por ejemplo, desde la carrera de Ingeniería Industrial se ha planteado la creación de dos centros de investigación: el Centro de Emprendimiento y Desarrollo Empresarial y el Instituto de Transferencia de Tecnología e Innovación. También se menciona el Centro de Investigación de la Quinua de Oruro, iniciado en 2010 con el objetivo de articular esfuerzos para el desarrollo tecnológico y mejora de la competitividad de la cadena quinua. Es un modelo exitoso que está conllevando diferentes actividades en líneas estratégicas como, por ejemplo, en la formación de recursos humanos.
También es una iniciativa relevante la que se está llevando a cabo, dentro de este contexto, con la creación del primer Parque Científico y Tecnológico de Oruro, promovido por el Gobierno de Oruro, la UTO y la FEPO, y que actualmente está en una fase inicial de construcción.

Por otro lado, la mayoría de las actividades de TT que se han realizado hasta ahora en la UTO se han desarrollado principalmente en los sectores agropecuario y minero (Entrevistado 5, entrevista 2012). En el sector agropecuario las investigaciones realizadas han estado muy ligadas a las actividades productivas de la región y se han realizado principalmente en el área rural, por lo que el contacto 
con el sector productivo ha sido fuerte $y$ estrecho, y los resultados han fluido de manera natural. En el sector minero la transferencia ha estado organizada. La UTO cuenta con dos unidades de servicio para realizar servicios relacionados con el análisis y desarrollo de químicos. En cuanto al resto de sectores no existen áreas de servicios destinadas a hacer TT. Con todo, se puede decir que en la UTO se está produciendo un cambio de mentalidad en cuanto a la TT.

Basados en la metodología cualitativa (Lockett et al., 2008; Padilla-Meléndez et al., 2013), se exponen las opiniones de los entrevistados, considerando los factores previamente señalados en el Cuadro 1.

En este sentido, los entrevistados señalan lo siguiente: Nosotros tenemos que estar en la industria, con los municipios, con la gobernación y es en la calle donde realmente nos necesitan (Entrevistado 5,entrevista 2012) y hay gente que está poco capacitada para dar un salto tecnológico. Puedo decirte que en algunos casos hay todavía empresarios antiguos que no usan la computadora o que no están en el mail cada día, entonces, es un cambio que se está logrando pero cuesta (Entrevistado 7, entrevista 2012)

Cada vez más hay una mayor conciencia de la importancia de vincularse con el entorno para conocer las necesidades existentes, y así, dirigir los trabajos de investigación al solvento de las mismas impidiendo que se queden "olvidados en un cajón". Hasta ahora, la investigación realizada en la UTO ha sido fruto prácticamente de iniciativas personales de docentes. El investigador decía quienes podían ser los beneficiarios de esa in- vestigación pero no se realizaba el contacto para verificar si realmente había una necesidad. Hasta ahora no había existido ese acercamiento entre universidad y beneficiarios, aunque esto está cambiando: Los proyectos futuros [...] tienen que tener un beneficiario directo. Esa transferencia tiene que ser más evidente (Entrevistado 1, entrevista 2012)

Gracias a este cambio de mentalidad, la UTO está abriendo sus puertas al exterior. Así, el Entrevistado 5,entrevista 2012 señala lo siguiente: Como universidad estamos relacionados con diferentes instituciones privadas, con entidades que promueven el desarrollo, con organizaciones de productores que hacen actividades productivas.

Este cambio también se ve reflejado en cuanto a que los docentes-investigadores de la UTO han empezado a dedicar parte de su tiempo de trabajo a contactar con instituciones externas: Dedico mucho tiempo, no es fácil captar empresas que quieran que investiguemos algo, aunque este último año sí que hemos tenido bastante requerimientos... (Entrevistado 4, entrevista 2012)

Las actividades de TT que se llevan a cabo en colaboración con las empresas son principalmente pasantías, es decir, prácticas de empresas donde los alumnos realizan o aplican sus tesis de grado. Así se señala: ...tenemos un seminario de grado de los quintos cursos que hacen planes de negocio. Los tesistas hacen sus planes de negocio para titularse $y$ para ello van a empresas pequeñas $y$ agarran su problemática y tratan de resolverla y dan alguna solución plasmada en un plan de negocios (Entrevistado 3, entrevista 2012) 
Además, aunque en menor medida, también se realizan convenios de colaboración con empresas para la prestación de servicios, principalmente de asesoría y gestión, o buscando material logístico que por su condición de pequeña empresa no posee y encuentran en la universidad:...la universidad tiene un equipo que no tenemos en las empresas así que hemos recurrido a ellos para que puedan colaborar, es decir, que nos manden su personal y sus equipos para que puedan hacer un análisis de determinados casos en la empresa (Entrevistado 9, entrevista 2012)

Muchas de estas últimas colaboraciones o ideas de investigación surgen de contactos informales. En Oruro, la TT es una actividad que ha comenzado a realizarse recientemente por lo que no existen instituciones que se encarguen de gestionar la oferta y la demanda entre la universidad y la empresa, es mediante contactos y reuniones informales como aparecen: Si alguien tiene interés llama directamente a la persona de contacto que conoce para establecer algún acuerdo. Nadie llama a la administración de la universidad para buscar algún trabajo conjunto o algo así. (Entrevistado 1, entrevista 2012)

Otra muestra de ello, es que en la UTO y en las empresas no hay establecidos métodos de evaluación de los resultados de la TT, salvo algunas empresas que evalúan el conocimiento adquirido por sus pasantes mediante cuestionarios al final de la pasantía. La mayoría basan su evaluación en percepciones u opiniones que se generan durante el proceso de la TT: No tenemos esa experiencia. En esta universidad se hace muy poca investigación, no hay una manera de como evaluarla. Al ser una actividad que recién está empezando nosotros mismo tenemos dificultad para evaluar las investigaciones (Entrevistado 1, entrevista 2012).

La UTO está siendo consciente de la importancia de su vinculación con el entorno, de hecho lo contempla como una línea de acción dentro del PDE 2009-2013, por ello está llevando a cabo una serie de iniciativas cuyo principal objetivo es el fomento de la investigación aplicada y el estrechamiento de su relación con el entorno.

En síntesis, se pueden resaltar elementos fundamentales basados en los resultados obtenidos. Con respecto a los factores que afectan a las actividades de TT entre la universidad y las empresas, especialmente a nivel individual, y el impacto de estas sobre el desarrollo regional. Al igual que en estudios anteriores (Gottwald et al., 2012; Lockett et al., 2009, McAdam et al., 2012; Vega-Jurado et al., 2007, 2009) este trabajo demuestra que se está produciendo un cambio en la mentalidad de los docentes/investigadores y directivos/empresarios sobre el rol de la universidad.

\subsection{Factores que afectan a la TT}

Del análisis realizado se extrae que la mayoría de los entrevistados perciben la TT como una obligación de la universidad. Cada vez más, docentes y empresarios, consideran el rol de la universidad como la generación de conocimientos y su transferencia hacia el exterior. Ahora la universidad no es sólo un agente de formación de profesionales, sino que ésta tiene un papel fundamental en la sociedad. El problema es que esta mentalidad todavía no se ha materializado en accio- 
nes concretas. No obstante, la actitud que muestran docentes y estudiantes de la universidad, como gerentes de empresas en el sector productivo es muy positiva hacia estas actividades. Así, se indica: La universidad desde mi punto de vista es una academia donde se debe de tener todos los conocimientos y después transferirlos (Entrevistado 4, entrevista 2012) y La TT es una actuación muy importante en la labor y la vida de ambas instituciones. Ahora estamos viendo que la universidad juega un papel importante aquí en las empresas y en las industrias (Entrevistado 8 , entrevista 2012).

Por otra parte los factores que afectan a la TT en Oruro se pueden dividir en oportunidades (para la Universidad y para las empresas) y barreras (burocracia, falta de financiación, falta de confianza y falta de profesionalidad).

\subsubsection{Factores que facilitan la TT: oportunidades}

En cuanto a las oportunidades para la Universidad, los beneficios que obtiene la universidad a la hora de realizar actividades de TT son varios. En primer lugar, realizar actividades de TT permite a la universidad desarrollarse y estar actualizada sobre la realidad, de manera que es más competitiva a la hora de dar formación a sus estudiantes. Así, se indica: La importancia de la TT relacionada con las empresas es de suma importancia porque es un factor de desarrollo no solo para la sociedad sino también para la universidad misma (Entrevistado 3, entrevista 2012).

En segundo lugar, es una forma de evaluarse como formadores viendo si realmente los estudiantes son capaces de afrontar la problemática que encuentran en el mundo laboral. A modo de ilustración, se señala: Yo veo que la universidad se beneficia porque le permite ver la capacidad de conocimiento que han transmitido a sus estudiantes en la práctica. De esta manera, pueden hacer una evaluación de la calidad de formación que están dando a sus estudiantes (Entrevistado 9, entrevista 2012).

Además, ayuda a la universidad a que tenga un mayor reconocimiento dentro de la sociedad: La TT le da espacio a la universidad para ser reconocida por la sociedad. La universidad tiene que cumplir ese rol, es decir, la universidad no solo forma profesionales o genera investigación, también hace vinculación (Entrevistado 5, entrevista 2012).

Por último, es una oportunidad para vincularse con su entorno: Los beneficios son enormes porque cuanto más se hace investigación, más se fortalece la educación, la formación de profesionales [...] son nuevas oportunidades de relacionarse con las universidades pero también con la empresa y con el gobierno, [...] (Entrevistado 1, entrevista 2012)

Por el lado de las oportunidades para las empresas, se señala que la TT es importante también por varias razones. En primer lugar, les permite renovarse y obtener mejores instrumentos para el desarrollo de su actividad ayudando a que sean más productivas y estén a la vanguardia en cuanto a la innovación dentro de su sector: Es totalmente importante porque yo creo que una empresa mejora cuando se renueva y tiene los mejores instrumentos para modernizarse... lo que necesitamos es re- 
tro-alimentación, que la universidad sea una especie de suero para la empresa privada (Entrevistado 8, entrevista 2012).

Las empresas del Departamento de Oruro son principalmente micro y pequeñas empresas que no cuentan con recursos económicos suficientes como para generar actividades de I+D. En este sentido, pueden encontrar en la universidad los medios y el personal cualificado para llevar a cabo estas actividades a un coste asumible: Las empresas si quieren estar a la vanguardia en su sector, en su rol productivo, entonces tienen que tener como aliado los que generan conocimiento $y$ en este caso es la universidad. (Entrevistado 2, entrevista 2012)

De esta manera, las empresas conseguirían ser más competitivas y tendrían la oportunidad de entrar en nuevos mercados: En lo que es innovación necesariamente tiene que haber una fuerte inyección de conocimiento en las empresas, y esto tiene que desarrollarse en las universidades y en los centros de investigación, lo cual se debe llevar a las empresas para que estas mejoren su competitividad, mejoren su calidad y realmente puedan competir con otras empresas. (Entrevistado 2, entrevista 2012)

Por tanto, se puede afirmar que las actividades de TT generan una serie de oportunidades y beneficios tanto para la universidad como para la empresa, permitiéndoles ser más competitivas y estar a la vanguardia en cuanto a innovación (Audretsch et al., 2012). Por parte de la universidad, las actividades de TT, proporcionan un continuo contacto con el entorno exterior que le favorece en cuanto a una actualización y evaluación continua de sus conocimientos, así como un ma- yor reconocimiento ante la sociedad (Siegel et al., 2003a, 2003b; Link et al. 2007); mientras que a la empresa le proporciona los medios y personal cualificado necesario para aumentar su competitividad en cuanto a innovación a un coste asequible (Decter et al., 2007).

\subsubsection{Oportunidades que limitan la TT: barreras}

En cuanto a las barreras, actualmente, se está produciendo un cambio de mentalidad entono a la TT pero todavía hay que entusiasmar y motivar a los estudiantes y docentes de la universidad para que lleven a cabo tareas de investigación ya que el número de investigadores todavía es muy reducido: En la UTO falta una verdadera cultura de interacción social, una vinculación, falta todavía esa conciencia, esa reflexión de que el tema de la TT tiene que ser absolutamente práctico. (Entrevistado 3, entrevista 2012)

La mayoría de los docentes señalan que existen más barreras que facilidades a la TT entre universidad y empresa. Esto se debe, como indica el Entrevistado 4, entrevista 2012, a: La principal causa de que no se genere más investigación es que el entorno no exige resultados fuertes en investigación porque no suelen aplicar dichos resultados.

Por otro lado, existen numerosas dificultades a la hora de llevar a cabo las labores de investigación dentro de la universidad: barreras burocráticas, falta de financiación, falta de confianza y falta de profesionalidad.

Las barreras burocráticas que existen se deben a la falta de regulación de estas actividades a todos los niveles. Así, 
se indica: Hay una falta de entendimiento acerca de la dirección en la que debe ir la investigación. (Entrevistado 1, entrevista 2012) y ... hace falta una política universitaria clara para la actividad investigadora o para la I+D y también otra a nivel de gobierno (Entrevistado 4, entrevista 2012)

La mayoría de los entrevistados mencionan la ley financial como un obstáculo a la investigación, principalmente porque no permite que los docentes de la universidad realicen otras labores dentro de carga horaria docente, y además, establece límites salariales a los docentes que realizan tareas de investigación.

La falta de financiación es, junto con las barreras burocráticas, la más citada entre los expertos. El principal problema que existe es que los fondos de los que dispone la universidad van destinados principalmente a la formación de profesionales. A pesar del aumento de fondos por la aportación del IDH, si se comparan los presupuestos de la UTO en investigación con el de otros países más avanzados, este sigue siendo muy bajo: Todo el flujo de recursos está destinado a financiar la actividad actual que es la formación de profesionales, hacer investigación aquí no está previsto, es una excepción (Entrevistado 1, entrevista 2012).

Por otro lado, las empresas, al ser en su mayoría de subsistencia, cuentan con muy pocos recursos y no se arriesgan a realizar inversiones a no ser que sea estrictamente necesario para la supervivencia de la empresa: Un factor importante es también el económico. Las empresas de alguna manera también son empresas de subsistencia pues no pueden invertir mucho en recursos tecnológicos (Entrevistado 6, entrevista 2012).
Además, a lo anterior, hay que sumarle el factor cultural. Existe una cultura muy tradicional en cuanto a la actividades de I+D lo que impide la aplicación de nuevos métodos para el desarrollo de su actividad. Por tanto, es necesaria una cultura más innovadora y una mayor visión de futuro: En el caso de las empresas hay algunos gerentes que son resistentes a la TT y algunas autoridades del mando intermedio. Falta emprendimiento y visión (Entrevistado 4, entrevista 2012).

Otra barrera es la falta de confianza sobre la universidad tanto por parte del sector productivo como de la toda la sociedad. Esto se debe en gran medida al desconocimiento, por ejemplo, se señala: Falta conocimiento por parte de muchos dirigentes sobre la importancia de la TTy los beneficios que reportan (Entrevistado 8, entrevista 2012).

En este sentido, es lógico que el desconocimiento genere falta de confianza y por tanto el apoyo institucional que reciben los pocos docentes o estudiantes que se dedican a la investigación sea escaso. Esto genera desconocimiento y por tanto desconfianza dentro del sector: La desconfianza del sector empresarial es muy latente ante la universidad [...], la universidad tiene que hacer los esfuerzos para revertir y ganar otra vez la confianza del sector productivo (Entrevistado 2, entrevista 2012).

Finalmente, la falta de profesionalidad, tanto por el lado empresarial como por la universidad, dificulta en muchas ocasiones que se puedan llevar a cabo actividades de TT: Muchas de nuestras empresas son muy informales. [...] y eso obviamente hace que algunos temas de la transferencia no funcionen (Entrevistado 6, entrevista 2012). 
Precisando en los factores que afectan la TT se ha distinguido entre aquellos que son oportunidades y motivan a la TT y aquellos que son barreras o dificultan la TT de modo similar a otros trabajos tal y como se ha expresado en el Cuadro 1 (Decter et al., 2007; Link et al., 2007; Padilla-Meléndez et al., 2013; Siegel et al., 2003a, 2003b, 2004). Entre los factores que motivan la TT, sigue apareciendo un mayor prestigio de la universidad dentro de la sociedad a través del reconocimiento en la labor de los docentes (Decter et al., 2007; Siegel et al., 2003a, 2003b) y un aumento de la relaciones y redes sociales de la universidad con el exterior (Gottwald et al., 2012; Link et al., 2007; Vega-Jurado et al. 2007) como factores determinantes de la TT. Por el contrario, entre los factores que dificultan la TT se encuentran: la barreras burocráticas (Gottwald et al., 2012; Siegel et al., 2004) debido al vacío legal existente en cuanto a la regulación de las actividades de investigación y TT (Vega-Jurado et al., 2007, 2009), la necesidad de reducir las horas de docencia y aumentar las de investigación (Siegel et al., 2004; Vega-Jurado et al., 2007, 2009), la falta de financiación (Decter et al., 2007; Gottwald et al., 2012; Siegel et al., 2003), la barrera cultural (Decter et al., 2007; Siegel et al., 2003a, 2003b, 2004; Vega-Jurado et al., 2007, 2009); y la falta de confianza entre los sectores (Vega-Jurado et al., 2007, 2009). Un elemento adicional que se ha encontrado tiene que ver con la falta de profesionalidad, motivada en parte porque no existe una regulación específica sobre el tema.

\subsection{Impacto sobre el desarrollo regional}

En el análisis de las entrevistas se obtiene como los entrevistados señalan que el principal beneficiario de las actividades de TT es la sociedad ya que se crea riqueza y empleo, es decir, desarrollo económico. Por lo que se puede afirmar que las actividades de TT entre la universidad y la empresa tiene un impacto de desarrollo a nivel regional: La sociedad es la que se va a ir beneficiando en la medida en que mejoren las condiciones de vida del lugar, que genere empleo, recursos humanos, [...] lo que se beneficia de todo esto al final es la región, el lugar, el departamento. (Entrevistado 2, entrevista 2012) y La TT nos ayuda a prepararnos, a enfrentarnos al futuro y nos ayuda definitivamente a desarrollar la zona, a crecer (Entrevistado 10, entrevista 2012).

Por tanto, todos los entrevistados definen las actividades de TT como algo positivo en términos de desarrollo. La mayoría de los mismos coincide en que es muy pronto para medir el resultado ya que las actividades de TT realizadas han sido muy pocas y muy recientes, por lo que habrá que esperar, pero todos creen que van por buen camino: Creemos que ha transcurrido muy poco tiempo para poder observar algún impacto decisivo (Entrevistado 1, entrevista 2012).

En cuanto al impacto de las actividades de TT en el desarrollo regional, este trabajo incide en que cada vez más el desarrollo económico está más vinculado a la capacidad de una región para adquirir y aplicar el conocimiento, lo que acentúa el 
papel de las universidades dentro de este proceso (Cornett, 2009; Lockett et al., 2009; McAdam et al., 2012; Teng, 2010). Este papel consiste en la creación de conocimientos y su transferencia al sector empresarial, lo que provoca una mejora en las empresas regionales y por tanto un aumento de las oportunidades de empleo de la región (Shaw y Allison, 1999). También se han encontrado evidencias de que el grado del impacto en el desarrollo regional va a depender de varios factores, no solo de las características de la propia universidad, sino también de los factores externos entre los que se encuentra envuelta (Smith y Bagchi-Sen, 2012). En este contexto, este trabajo coincide con Huggins et al. (2012) en que la red de contactos de organizaciones con las que la universidad realiza actividades de TT afecta a la capacidad regional de innovación y por tanto a la competitividad regional. Por último, este estudio es un claro ejemplo en el que se puede observar que para conocer el impacto de las actividades de TT sobre el desarrollo regional hace falta tiempo y paciencia (Bercovitz y Feldmann, 2006) debido al contexto donde se ha realizado, en el cual estas actividades todavía son incipientes.

\section{Conclusiones}

Los principales resultados encontrados reflejan que las actividades de TT entre la Universidad Técnica de Oruro (UTO) y las empresas del Departamento de Oruro son todavía muy incipientes.

En cuanto a las actividades de TT que se han llevado a cabo en el Departamento de Oruro, se han producido principalmente en el sector agropecuario y $\mathrm{mi}$ - nero, debido a que son los dos sectores más explotados de la región y los que más demanda de nuevos conocimiento generan. En cuanto al tipo de actividades, las pasantías o prácticas en empresas son las que más se realizan. También se llevan a cabo convenios de colaboración con empresas para la prestación de servicios, principalmente de asesoría y gestión de empresas, y para la búsqueda de material logístico debido a que la mayoría de las empresas, por su condición de pequeñas o medianas, no poseen y fácilmente pueden encontrar en la universidad. Con respecto a los factores que afectan a la TT se distingue entre oportunidades y riesgos.

Las oportunidades para la universidad son: un mayor desarrollo interno al permitirle una actualización de los conocimientos que transfiere a sus estudiantes, de esta manera los docentes pueden evaluarse como formadores al ver si sus alumnos son capaces de enfrentarse con la problemática del mundo real; un mayor reconocimiento y prestigio dentro de la sociedad; y la oportunidad de vincularse con su entorno. Las oportunidades que genera a las empresas son: les permite la renovación y obtención de mejores instrumentos para el desarrollo de su actividad ayudando a que sean más productivas y estén a la vanguardia en cuanto a la innovación dentro de su sector, aquellas que no cuenten con recursos económicos suficientes como para tener un departamento de I+D puede externalizarlo en la universidad a unos costos asumibles, y como resultado de estos dos últimos, las empresas consiguen ser más competitivas y por tanto tienen mayores oportunidades de entrar en nuevos mercados. 
Entre los riesgos de realizar actividades de TT para la universidad y la empresa destacan las barreras burocráticas; estas se deben a la falta de una regulación de las actividades de investigación y transferencia a todos los niveles. Además, hay que sumarle que no existe todavía un entendimiento claro acerca de la dirección en la que debe ir la investigación y la falta de incentivos a los docentes ahora hora de realizar tareas de investigación. Este vacío legal provoca que la mayoría de las relaciones entre la universidad y las empresas tenga carácter informal. De igual forma, aparece la falta de financiación. Ésta, junto con las barreras burocráticas, son las más destacadas por los docentes. Otra barrera es la falta de confianza depositada en la universidad tanto por el sector productivo como por toda la sociedad. Esto se debe principalmente a la falta de conocimiento por parte de muchos dirigentes sobre la importancia de la TT y los beneficios que reportan. Otro factor que impide que se puedan llevar a cabo actividades de TT es la falta de profesionalidad en general.

Por otro lado, se reconoce que el impacto de estas actividades sobre el desarrollo regional es importante. Así, todos los entrevistados coinciden en que es una herramienta muy positiva pero que todavía no se ha materializado. Se ha encontrado una actitud muy positiva hacia la realización de esta actividades, el problema que se plantea principalmente son las características del contexto. Además, al ser las actividades de TT realizadas en Oruro muy incipientes todavía no se ha podido medir el impacto que estas han podido tener sobre el desarrollo regional.
De estas conclusiones cabe extraer implicaciones a diversos niveles. A nivel político, es necesario involucrar a las autoridades nacionales, a los departamentales y a los órganos reguladores de la actividad universitaria. En este sentido, es necesario establecer una política clara en cuanto a las labores de investigación dentro de la universidad. Esto conlleva la descentralización de las estructuras de funcionamiento de los docentes para dar una mayor autonomía en cuanto a las actividades de investigación y establecer una estructura de incentivos adecuada para recompensar los esfuerzos de emprendimiento académico, tales como: disminución de hora de docencia por carga investigadora, mayor reconocimiento dentro de la organización e incentivos económico, entre otros.

A nivel organizativo, es preciso el establecimiento de un servicio centralizado de personal con experiencia que regule y gestione el funcionamiento de estas actividades. Es decir, que actúe de intermediario poniendo en contacto la demanda con la oferta de investigación de manera que todos los trabajos que se realicen dentro de la universidad sean útiles para el sector productivo, y además, se encargue de la gestión de dichos resultados. A nivel individual, resulta claro que si se quiere promover la TT entre docentes/investigadores y empresarios/directivos, es preciso clarificar los vacíos legales señalados, así como mejorar, sustancialmente el reconocimiento institucional y social de estas actividades.

Finalmente, como todo trabajo de investigación, este no está exento de limitaciones. En primer lugar, se trata de un trabajo exploratorio. Otros estudios cuan- 
titativos podrían arrojar más luz y por tanto conclusiones más confirmatorias sobre el efecto de una adecuada política de TT en el desarrollo regional. En segundo lugar, se trata de un estudio realizado en un contexto particular, cual es la UTO y el Departamento de Oruro. Este estudio podría extrapolarse a nivel nacional, comparando los sistemas de transferencia y tecnología establecidos en otras regiones de manera que sirvan como ejemplo aquellas regiones menos desarrolladas en este aspecto, y así pasar de un desarrollo regional al desarrollo de toda la nación, lo cual señala una segunda línea de investigación futura. Por último, es necesario comparar esta realidad con otros contextos a nivel internacional, no ya solo a nivel de países en vías de desarrollo sino con países más avanzados.

\section{Referencias Bibliográficas}

Adler, Paul S. y Kwon, Seok-Woo (2002). Social capital: Prospects for a new concept. The Academy of Management Review, Vol. 27, No. 1, pp. 17-40.

Alvesson, Mats y Sköldberg, Kaj. (2000). Reflexive Methodology: New Vistas for Qualitative Research. London: Sage.

Audretsch, David B.; Hüsbeck, Marcel y Lehmann, Erik E. (2012). Regional competitiveness, university spillovers, and entrepreneurial activity. Small Business Economics, Vol. 39, No 3, pp. 587-601.

Bansal, Pratima y Corley, Kevin (2012). From the Editors Publishing in AMJ-Part 7: What's Different about Qualitative Research? Academy of Management Journal, Vol. 55, No. 3, pp. 509-513.
Bercovitz, Jannet y Feldmann, Maryann (2006). Entrepreneurial Universities and Technology Transfer: A Conceptual Framework for Understanding Knowledge-Based Economic Development. Journal of Technology Transfer, Vol. 31, No. 1, pp. 175-188.

CEPAL y Naciones Unidas (2012). Apoyando a las pymes: Políticas de fomento en América Latina y el Caribe. Disponible en: http://www.eclac.cl/publicaciones/xml/0/45410/LCR.2180.pdf (accedido el 9/11/2012)

Chesbrough, Henry (2003). Open Innovation: The New Imperative for Creating and Profiting from Technology. Cambridge, MA: Harvard Business School Publishing.

Chesbrough, Henry; Vanhaverbeke, Wim y West, Joel (2006). Open Innovation: Researching a New Paradigm. Oxford: Oxford University Press.

Cornett, Andreas P. (2009). Aims and strategies in regional innovation and growth policy: A Danish perspective. Entrepreneurship \& Regional Development, Vol. 21, No. 4, pp. 399-420.

Debackere, Koenraad y Veugelers, Reinhilde (2005). The role of academic technology transfer organizations in improving industry science links. Research Policy, Vol. 34, No. 3, pp. 321-342.

Decter, Moira; Bennett David y Leseure, Michel (2007). University to business technology transfer: UK and USA comparisons. Technovation, Vol. 27, No. 3, pp. 145-155.

D’Este, Pablo y Perkmann, Markus (2011). Why do academics engage with industry? The entrepreneurial university and individual motivations. Journal of Technology Transfer, Vol. 36, No. 3, pp. 316-339.

Etzkowitz, Henry (2002). MIT and the Rise of Entrepreneurial Science. Routledge, London. 
Etzkowitz, Henry (2003). Research groups as 'quasi-firms': The invention of the entrepreneurial university. Research Policy, Vol. 32, No. 1, pp. 109-121.

Etzkowitz, Henry; Webster, Andrew; Gebhardt, Christiane y Cantisano-Terra, Branca Regina (2000), The future of the university and the university of the future: Evolution of ivory tower to entrepreneurial paradigm. Research Policy, Vol. 29, No. 2, pp. 313-330.

Gassmann, Oliver; Enkel, Ellen y Chesbrough, Henry (2010). The future of open innovation. R\&D Management, Vol. 40, No. 3, 213-221.

Gobierno Autónomo Departamental de Oruro (2011). Plan de Desarrollo Departamental. Disponible en: http://bibliocomunidad.com/web/libros/PDD\%200RURO\%202011\%20-\%202015.pdf (accedido el 19/11/2012)

Gottwald, Julia; Buch, Franziska y Giesecke, Kira (2012). Understanding the role of universities in technology transfer in the renewable energy sector in Bolivia. Management of Environmental Quality: An International Journal, Vol. 23, No. 3, pp. 291-299.

Huggins, Robert, Johnston, Andrew y Stride, Chris (2012). Knowledge networks and universities: Locational and organizational aspects of knowledge transfer interactions. Entrepreneurship \& Regional Development, Vol. 24, No. 7-8, pp. 475-502.

Instituto Nacional de Estadística de Bolivia (2010). Estadística e Indicadores Económicos y Sociodemográdicos de Bolivia. INEB. Disponible en: http://www.ine.gob.bo/pdf/Bo_Es_Na /BEN_2010_1.pdf (accedido el $5 / 11 / 2012$ )

Instituto Nacional de Estadística de Bolivia (2011). Estadística Socioeconómicas del Departamento de Oruro.
INEB. Disponible en: http://www.ine. gob.bo/pdf/Est_Dptales/EN_2011_1. pdf (accedido el 29/11/2012).

Link, Albert N., Siegel, Donald S. y Bozeman, Barry (2007). An empirical analysis of the propensity of academics to engage in informal university technology transfer. Industrial and Corporate Change, Vol. 16, No. 4, pp. 641-655.

Lockett, Nigel; Cave, Frank; Kerr, Ron y Robinson, Sarah (2009). The influence of co-location in higher education institutions on small firms' perspectives of knowledge transfer. Entrepreneurship \& Regional Development, Vol. 21, No. 3, pp. 265-283.

Lockett, Nigel; Kerr, Ron y Robinson, Sarah (2008), Multiple Perspectives on the Challenges for Knowledge Transfer between HEls and Industry. International Small Business Journal, Vol. 26, No. 6, pp. 661-681.

McAdam, Rodney; Keogh, William, Galbraith, Brendan y Laurie, Don (2005). Defining and improving technology transfer business and management processes in university innovation centers. Technovation, Vol. 25 , No. 12 , pp. 1418-1429.

McAdam, Rodney; Miller, Kristel, McAdam, Maura y Teague, Sinead (2012). The development of University Technology Transfer stakeholder Relationships at a regional level: Lessons for the future. Technovation, Vol. 32, No. 1, pp. 57-67.

O'Shea; Rory, Allen, Thomas J., O'Gorman, Colm y Roche, Frank (2004). University and Technology Transfer: A Review of Academic Entrepreneurship Literature. Irish Journal of Management, Vol. 25, No. 2, pp. 11-29.

Padilla-Meléndez, Antonio y Garrido-Moreno, Aurora (2012). Open innovation in universities: What motivates researchers 
to engage in knowledge transfer exchanges? International Journal of Entrepreneurial Behaviour \& Research, Vol. 18, No. 4, pp. 417-439.

Padilla-Meléndez, Antonio; Del Águila-Obra; Ana Rosa y Lockett, Nigel (2013). Shifting sands: Regional perspectives on the role of social capital in supporting open innovation through knowledge transfer and exchange with small and medium-sized enterprises. International Small Business Journal, Vol. 31, No. 3, pp. 295-317.

Philpott, Kevin; Dooley, Lawrence; O’Reilly, Caroline y Lupton, Gary (2011). The entrepreneurial university: Examining the underlying academic tensions. Technovation, Vol. 31, No. 4, pp. 161170.

Quiñones, Ainoa y Tezanos, Sergio (2011). Ayuda Oficial al Desarrollo CientíficoTecnológica: una Evaluación Macroeconómica de la Distribución Geográfica y Sectorial. Revista de Economía Mundial, Vol. 29, pp. 151-177.

RICYT (2012). Indicadores de Ciencia y Tecnología en América Latina. Disponible en: http://www.ricyt.org/index.php?option=com_content\&view=article\&id=149\&ltemid=3 (accedido el 5/11/2012)

Rogers, Everett M. (1983). The Diffusion of Innovation. New York: The Free Press.

Shaw, Julie Keane y Allison, Janelle (1999). The intersection of the learning region and local and regional economic development: Analysing the role of higher education. Regional Studies, Vol. 33, No. 9, pp. 896-915.

Siegel, Donald S.; Waldman, David A.; Atwater, Leanne E. y Link, Albert N. (2004). Toward a model of the effective transfer of scientific knowledge from academicians to practitioners: qualitative evidence from the commercialization of university technologies. Journal of Engineering and Technology Management, Vol. 21, No 1-2, pp. 115142.

Siegel, Donald S.; Waldman, David A.; Atwater, Leanne E. y Link, Albert N. (2003). Commercial knowledge transfers from universities to firms: improving the effectiveness of University-industry collaboration. Journal of High Technology Management Research, Vol. 14, No. 1, pp. 111-133.

Siegel, Donald S.; Waldman, David y Link, Albert (2003). Assessing the impact of organizational practices on the relative productivity of university technology transfer offices: An exploratory study. Research Policy, Vol. 32, No. 1 , pp. 27-48.

Silverman, David (2000). Doing Qualitative Research: A Practical Handbook. Thousand Oaks, CA: Sage.

Smith, Hewlen Lawton y Bagchi-Sen, Sharmistha (2012). The research university, entrepreneurship and regional development: Research propositions and current evidence. Entrepreneurship \& Regional Development: An International Journal, Vol. 24, No. 5-6, pp. 383-404.

Taylor, Steven J. y Bogdan, Robert (2000). Introduction to qualitative research methods: the search for meanings. Wiley.

Teng, H. (2010). University-Industry Technology Transfer: Framework and Constraints. Journal of Sustainable Development, Vol. 3, No. 2, pp. 296300.

Universidad Técnica de Oruro (UTO) (2009). Plan de Desarrollo Estratégico 2009-2013. Disponible en: http://www.uto.edu.bo/documentos/menu1/PDE-29julio.pdf (accedido el 23/11/2012). 
Vega-Jurado, Jaider M.; Fernández-de-Lucio, Ignacio y Huanca-López, Ronald (2007), ¿La Relación UniversidadEmpresa en América Latina: Apropiación Incorrecta de Modelos Foráneos?. Journal of Technology Management, Vol.2, No. 2, pp. 97-109.

Vega-Jurado, Jaider M.; Huanca-López, Ronald; Fernández-de-Lucio, Ignacio y Manjarres, Liney A. (2009), Papel De La I+D En La Relación Universidad Empresa: Una Visión Desde El Sur. Revista Iberoamericana de Ciencia, Tecnología, Sociedad e Innovación, No. Extraordinario, pp. 223-251.

Yin, Robert K. (2011), Qualitative Research from Start to Finish. New York and London: The Guilford Press.

\section{Entrevistas}

Entrevistado 1. Responsable del área de Ingeniería y Tecnología de la UTO. Departamento de Investigación Científica y Tecnológica (UTO)

Entrevistado 2. Responsable del Proyecto de Incubadora de Empresas de Bases Tecnológicas de la UTO y Profesor e Investigador de la UTO. Carrera de In- geniería Industrial de la Facultad Nacional de Ingeniería (UTO).

Entrevistado 3. Profesor e Investigador de la UTO. Carrera de Administración de Empresas de la Facultad de Ciencias Económicas, Financieras y Administrativas (UTO).

Entrevistado 4. Responsable del Proyecto de Incubadora de Empresas de Bases Tecnológicas y Profesor e Investigador de la UTO. Carrera de Ingeniería Industrial de la Facultad Nacional de Ingeniería (UTO)

Entrevistado 5. Director de Investigación Científica y Tecnológica de la UTO. Departamento de Investigación Científica y Tecnológica (UTO).

Entrevistado 6. Profesor e Investigador de la UTO. Carrera de Ingeniería de Sistemas de la Facultad Nacional de Ingeniería (UTO).

Entrevistado 7. Secretario Ejecutivo. Federación de Empresarios Privados de Oruro (FEPO).

Entrevistado 8. Director de PYME. Sector comercio.

Entrevistado 9. Director de PYME. Sector industrial.

Entrevistado 10. Director de PYME. Sector industrial (construcción). 